\title{
Differential Subordination with Generalized Derivative Operator of Analytic Functions
}

\author{
Entisar El-Yagubi and Maslina Darus \\ School of Mathematical Sciences, Faculty of Science and Technology, Universiti Kebangsaan Malaysia, 43600 Bangi, \\ Selangor Darul Ehsan, Malaysia
}

Correspondence should be addressed to Maslina Darus; maslina@ukm.edu.my

Received 26 January 2014; Accepted 3 August 2014; Published 20 August 2014

Academic Editor: Annamaria Barbagallo

Copyright (C) 2014 E. El-Yagubi and M. Darus. This is an open access article distributed under the Creative Commons Attribution License, which permits unrestricted use, distribution, and reproduction in any medium, provided the original work is properly cited.

Motivated by generalized derivative operator defined by the authors (El-Yagubi and Darus, 2013) and the technique of differential subordination, several interesting properties of the operator $\mathscr{D}_{\lambda_{1}, \lambda_{2}, \delta}^{m, b}$ are given.

\section{Introduction}

Let $\mathscr{A}$ denote the class of functions of the form

$$
f(z)=z+\sum_{n=2}^{\infty} a_{n} z^{n}
$$

which are analytic in the open unit disk $\mathbb{U}=\{z: z \in \mathbb{C},|z|<$ $1\}$.

Also let $\mathcal{S}$ be the the subclass of $\mathscr{A}$ consisting of all functions which are univalent in $\mathbb{U}$. We denote by $\mathcal{S}^{*}(\alpha)$ and $\mathscr{C}(\alpha)(0 \leq \alpha<1)$ the familiar subclasses of $\mathscr{A}$ consisting of functions which are, respectively, starlike of order $\alpha$ and convex of order $\alpha$ in $\mathbb{U}$ :.

$$
\begin{aligned}
& \mathcal{S}^{*}(\alpha)=\left\{f \in \mathscr{A}: \mathfrak{R}\left\{\frac{z f^{\prime}(z)}{f(z)}\right\}>\alpha, z \in \mathbb{U}\right\}, \\
& \mathscr{C}(\alpha)=\left\{f \in \mathscr{A}: \mathfrak{R}\left\{1+\frac{z f^{\prime \prime}(z)}{f^{\prime}(z)}\right\}>\alpha, z \in \mathbb{U}\right\} .
\end{aligned}
$$

Let $\mathscr{H}(\mathbb{U})$ be the class of holomorphic function in unit disk $\mathbb{U}=\{z: z \in \mathbb{C},|z|<1\}$. For $a \in \mathbb{C}$ and $n \in \mathbb{N}$ we let

$$
\begin{aligned}
\mathscr{H}[a, n]=\{f & \in \mathscr{H}(\mathbb{U}), f(z)=a+a_{n} z^{n} \\
& \left.+a_{n+1} z^{n+1}+\cdots\right\}, \quad(z \in \mathbb{U}) .
\end{aligned}
$$

Let two functions given by $f(z)=\sum_{n=2}^{\infty} a_{n} z^{n}$ and $g(z)=$ $\sum_{n=2}^{\infty} b_{n} z^{n}$ be analytic in $\mathbb{U}$. Then the Hadamard product (or convolution) $f * g$ of the two functions $f, g$ is defined by

$$
f(z) * g(z)=z+\sum_{n=2}^{\infty} a_{n} b_{n} z^{n} .
$$

Recall that the function $f$ is subordinate to $g$ if there exists the Schwarz function $\omega$, analytic in $\mathbb{U}$, with $\omega(0)=0$ and $|\omega(z)|<1$ such that $f(z)=g(\omega(z)), z \in \mathbb{U}$. We denote this subordination by $f(z) \prec g(z)$. If $g(z)$ is univalent in $\mathbb{U}$, then the subordination is equivalent to $f(0)=g(0)$ and $f(\mathbb{U}) \subset g(\mathbb{U})$.

Let $\psi: \mathbb{C}^{3} \times \mathbb{U} \rightarrow \mathbb{C}$ and $h$ be univalent in $\mathbb{U}$. If $p$ is analytic in $\mathbb{U}$ and satisfies the (second order) differential subordination

$$
\psi\left(p(z), z p^{\prime}(z), z^{2} p^{\prime \prime}(z) ; z\right) \prec h(z), \quad(z \in \mathbb{U}),
$$

then $p$ is called a solution of the differential subordination.

The univalent function $q$ is called a dominant of the solutions of the differential subordination, or more simply a dominant, if $p \prec q$ for all $p$ satisfying (5).

A dominant $\tilde{q}$ that satisfies $\tilde{q} \prec q$ for all dominants $q$ of (5) is said to be the best dominant of (5) (note that the best dominant is unique up to a rotation of $\mathbb{U}$ ).

In order to prove the original results we need the following lemmas. 
Lemma 1 (see [1]). Let $h$ be a convex function with $h(0)=a$ and let $\gamma \in \mathbb{C}-\{0\}$ be a complex number with $\mathfrak{R}\{\gamma\} \geq 0$. If $p \in H[a ; n]$ and

$$
p(z)+\frac{z p^{\prime}(z)}{\gamma} \prec h(z), \quad(z \in \mathbb{U}),
$$

then

$$
p(z)<q(z)<h(z), \quad(z \in \mathbb{U}),
$$

where

$$
q(z)=\frac{\gamma}{n z^{\gamma / n}} \int_{0}^{z} h(t) t^{(\gamma / n)-1} d t, \quad(z \in \mathbb{U}) .
$$

The function $q$ is convex and is the best dominant.

Lemma 2 (see [2]). Let $g$ be a convex function in $\mathbb{U}$ and let

$$
h(z)=g(z)+n \alpha z g^{\prime}(z),
$$

where $\alpha>0$ and $n$ is a positive integer. If

$$
p(z)=g(0)+p_{n} z^{n}+p_{n+1} z^{n+1}+\cdots, \quad(z \in \mathbb{U})
$$

is analytic in $\mathbb{U}$ and

$$
p(z)+\alpha z p^{\prime}(z)<h(z), \quad(z \in \mathbb{U}),
$$

then

$$
p(z) \prec g(z),
$$

and this result is sharp.

Lemma 3 (see [3]). Let $f \in \mathscr{A}$; if

$$
\Re\left\{1+\frac{z f^{\prime \prime}(z)}{f^{\prime}(z)}\right\}>-\frac{1}{2},
$$

then

$$
\frac{2}{z} \int_{0}^{z} f(t) d t, \quad(z \in \mathbb{U}, z \neq 0)
$$

belongs to the class of convex functions.

We now state the following generalized derivative operator [4]:

$$
\begin{aligned}
& \mathscr{D}_{\lambda_{1}, \lambda_{2}, \delta}^{m, b} f(z) \\
& \quad=z+\sum_{n=2}^{\infty}\left[\frac{1+\left(\lambda_{1}+\lambda_{2}\right)(n-1)+b}{1+\lambda_{2}(n-1)+b}\right]^{m} \mathscr{C}(\delta, n) a_{n} z^{n},
\end{aligned}
$$

where $\lambda_{2} \geq \lambda_{1} \geq 0, \mathscr{C}(\delta, n)=(\delta+1)_{n-1} /(n-1)$ !, for $\delta, m, b \in \mathbb{N}_{0}=\{0,1,2, \ldots\}$, and $(x)_{n}$ is the Pochhammer symbol defined by

$$
\begin{aligned}
(x)_{n} & =\frac{\Gamma(x+n)}{\Gamma(x)} \\
& = \begin{cases}1, & n=0, \\
x(x+1) \cdots(x+n-1), & n=\{1,2,3, \ldots\} .\end{cases}
\end{aligned}
$$

Here $\mathscr{D}_{\lambda_{1}, \lambda_{2}, \delta}^{m, b} f(z)$ can also be written in terms of convolution as

$$
\mathscr{D}_{\lambda_{1}, \lambda_{2}, \delta}^{m, b} f(z)=\mathscr{D}_{\lambda_{1}, \lambda_{2}, \delta}^{m, b} f(z) * \mathscr{D}_{\lambda_{2}}^{m, b}(z) .
$$

To prove our results, we need the following inclusion relation:

$$
\begin{aligned}
(1+b) & \mathscr{D}_{\lambda_{1}, \lambda_{2}, \delta}^{m+1, b} f(z) \\
= & \left(1-\left(\lambda_{1}+\lambda_{2}\right)+b\right)\left(\mathscr{D}_{\lambda_{1}, \lambda_{2}, \delta}^{m, b} f(z) * \varphi_{\lambda_{2}}^{b}(z)\right) \\
& +\left(\lambda_{1}+\lambda_{2}\right) z\left(\mathscr{D}_{\lambda_{1}, \lambda_{2}, \delta}^{m, b} f(z) * \varphi_{\lambda_{2}}^{b}(z)\right)^{\prime},
\end{aligned}
$$

where $\varphi_{\lambda_{2}}^{b}(z)$ is analytic function given by $\varphi_{\lambda_{2}}^{b}(z)=z+$ $\sum_{n=2}^{\infty}\left(z^{n} /\left(1+\lambda_{2}(n-1)+b\right)\right)$.

\section{Main Results}

In the present paper, we will use the method of differential subordination to derive certain properties of generalised derivative operator $\mathscr{D}_{\lambda_{1}, \lambda_{2}, \delta}^{m, b} f(z)$. Note that differential subordination has been studied by various authors, and here we follow similar works done by Oros [5] and G. Oros and G. I. Oros [6].

Definition 4. For $\lambda_{2} \geq \lambda_{1} \geq 0, \delta, m, b \in \mathbb{N}_{0}$, and $0 \leq \alpha<1$, let $\mathscr{R}_{\lambda_{1}, \lambda_{2}, \delta}^{m, b}(\alpha)$ denote the class of functions $f \in \mathscr{A}$ which satisfy the condition

$$
\mathfrak{R}\left(\mathscr{D}_{\lambda_{1}, \lambda_{2}, \delta}^{m, b} f(z)\right)^{\prime}>\alpha, \quad(z \in \mathbb{U}) .
$$

Also, let $\mathscr{K}_{\lambda_{1}, \lambda_{2}, \delta}^{m, b}(\beta)$ denote the class of functions $f \in \mathscr{A}$ which satisfy the condition

$$
\Re\left(\mathscr{D}_{\lambda_{1}, \lambda_{2}, \delta}^{m, b} f(z) * \varphi_{\lambda_{2}}^{b}(z)\right)^{\prime}>\beta, \quad(z \in \mathbb{U}) .
$$

Remark 5. It is clear that $\mathscr{R}_{\lambda_{1}, 0,0}^{1,0}(\alpha) \equiv \mathscr{R}\left(\lambda_{1}, \alpha\right)$, and the class of functions $f \in \mathscr{A}$ satisfy

$$
\Re\left(\lambda_{1} z f^{\prime \prime}(z)+f^{\prime}(z)\right)>\alpha, \quad(z \in \mathbb{U}),
$$

studied by Ponnusamy [7] and others.

Theorem 6. Let

$$
h(z)=\frac{1+(2 \alpha-1) z}{1+z}, \quad(z \in \mathbb{U}),
$$

be convex in $\mathbb{U}$, with $h(0)=1$ and $0 \leq \alpha<1$.

If $\lambda_{2} \geq \lambda_{1} \geq 0, \delta, m, b \in \mathbb{N}_{0}$, and $f \in \mathscr{A}$ and satisfies the differential subordination

$$
\left(\mathscr{D}_{\lambda_{1}, \lambda_{2}, \delta}^{m+1, b} f(z)\right)^{\prime}<h(z), \quad(z \in \mathbb{U}),
$$

then

$$
\begin{aligned}
\left(\mathscr{D}_{\lambda_{1}, \lambda_{2}, \delta}^{m, b} f(z) * \varphi_{\lambda_{2}}^{b}(z)\right)^{\prime} & \\
\quad & <q(z) \\
\quad & =2 \alpha-1+\frac{2(1-\alpha)(1+b)}{\left(\lambda_{1}+\lambda_{2}\right) z^{(1+b) /\left(\lambda_{1}+\lambda_{2}\right)}} \sigma\left(\frac{1+b}{\lambda_{1}+\lambda_{2}}\right),
\end{aligned}
$$


where $\sigma$ is given by

$$
\sigma(x)=\int_{0}^{z} \frac{t^{x-1}}{1+t} d t, \quad(z \in \mathbb{U}) .
$$

The function $q$ is convex and is the best dominant.

Proof. By differentiating (18), with respect to $z$, we obtain

$$
\begin{aligned}
& (1+b)\left(\mathscr{D}_{\lambda_{1}, \lambda_{2}, \delta}^{m+1, b} f(z)\right)^{\prime} \\
& =(1+b)\left(\mathscr{D}_{\lambda_{1}, \lambda_{2}, \delta}^{m, b} f(z) * \varphi_{\lambda_{2}}^{b}(z)\right)^{\prime} \\
& \quad+\left(\lambda_{1}+\lambda_{2}\right) z\left(\mathscr{D}_{\lambda_{1}, \lambda_{2}, \delta}^{m, b} f(z) * \varphi_{\lambda_{2}}^{b}(z)\right)^{\prime \prime} .
\end{aligned}
$$

Using (26) in (23), the differential subordination (23) becomes

$$
\begin{aligned}
& \left(\mathscr{D}_{\lambda_{1}, \lambda_{2}, \delta}^{m, b} f(z) * \varphi_{\lambda_{2}}^{b}(z)\right)^{\prime} \\
& \quad+\frac{\left(\lambda_{1}+\lambda_{2}\right)}{1+b} z\left(\mathscr{D}_{\lambda_{1}, \lambda_{2}, \delta}^{m, b} f(z) * \varphi_{\lambda_{2}}^{b}(z)\right)^{\prime \prime} \\
& \quad \prec h(z)=\frac{1+(2 \alpha-1) z}{1+z} .
\end{aligned}
$$

Let

$$
\begin{aligned}
& p(z) \\
& =\left(\mathscr{D}_{\lambda_{1}, \lambda_{2}, \delta}^{m, b} f(z) * \varphi_{\lambda_{2}}^{b}(z)\right)^{\prime} \\
& =\left(z+\sum_{n=2}^{\infty}\left(\frac{\left(1+\left(\lambda_{1}+\lambda_{2}\right)(n-1)+b\right)^{m}}{\left(1+\lambda_{2}(n-1)+b\right)^{m+1}}\right) \mathscr{C}(\delta, n) a_{n} z^{n}\right)^{\prime} \\
& =1+p_{1} z+p_{2} z^{2}+\cdots, \quad(p \in \mathscr{H}[1,1], z \in \mathbb{U}) .
\end{aligned}
$$

Using (28) in (27), the differential subordination becomes

$$
p(z)+\frac{\left(\lambda_{1}+\lambda_{2}\right)}{1+b} z p^{\prime}(z) \prec h(z)=\frac{1+(2 \alpha-1) z}{1+z} .
$$

By using Lemma 1, we have

$$
\begin{aligned}
p(z) \prec & q(z) \\
= & \frac{1+b}{\left(\lambda_{1}+\lambda_{2}\right) z^{(1+b) /\left(\lambda_{1}+\lambda_{2}\right)}} \int_{0}^{z} h(t) t^{\left((1+b) /\left(\lambda_{1}+\lambda_{2}\right)\right)-1} d t \\
= & \frac{1+b}{\left(\lambda_{1}+\lambda_{2}\right) z^{(1+b) /\left(\lambda_{1}+\lambda_{2}\right)}} \\
& \times \int_{0}^{z}\left(\frac{1+(2 \alpha-1) t}{1+t}\right) t^{\left((1+b) /\left(\lambda_{1}+\lambda_{2}\right)\right)-1} d t \\
= & 2 \alpha-1+\frac{2(1-\alpha)(1+b)}{\left(\lambda_{1}+\lambda_{2}\right) z^{(1+b) /\left(\lambda_{1}+\lambda_{2}\right)}} \sigma\left(\frac{1+b}{\lambda_{1}+\lambda_{2}}\right),
\end{aligned}
$$

where $\sigma$ is given by (25); that is,

$$
\begin{aligned}
& \left(\mathscr{D}_{\lambda_{1}, \lambda_{2}, \delta}^{m, b} f(z) * \varphi_{\lambda_{2}}^{b}(z)\right)^{\prime} \\
& \quad \prec q(z) \\
& \quad=2 \alpha-1+\frac{2(1-\alpha)(1+b)}{\left(\lambda_{1}+\lambda_{2}\right) z^{(1+b) /\left(\lambda_{1}+\lambda_{2}\right)}} \sigma\left(\frac{1+b}{\lambda_{1}+\lambda_{2}}\right) .
\end{aligned}
$$

The function $q$ is convex and is the best dominant. The proof is complete.

Theorem 7. If $\lambda_{2} \geq \lambda_{1} \geq 0, \delta, m, b \in \mathbb{N}_{0}$, and $0 \leq \alpha<1$, then one has

$$
\mathscr{R}_{\lambda_{1}, \lambda_{2}, \delta}^{m+1, b}(\alpha) \subset \mathscr{K}_{\lambda_{1}, \lambda_{2}, \delta}^{m, b}(\beta)
$$

where

$$
\beta=2 \alpha-1+\frac{2(1-\alpha)(1+b)}{\left(\lambda_{1}+\lambda_{2}\right)} \sigma\left(\frac{1+b}{\lambda_{1}+\lambda_{2}}\right),
$$

and $\sigma$ is given by (25).

Proof. Let $f \in \mathscr{R}_{\lambda_{1}, \lambda_{2}, \delta}^{m+1, b}(\alpha)$, and then from (19) we have

$$
\mathfrak{R}\left(\mathscr{D}_{\lambda_{1}, \lambda_{2}, \delta}^{m+1, b} f(z)\right)^{\prime}>\alpha, \quad(z \in \mathbb{U})
$$

which is equivalent to

$$
\left(\mathscr{D}_{\lambda_{1}, \lambda_{2}, \delta}^{m+1, b} f(z)\right)^{\prime} \prec h(z)=\frac{1+(2 \alpha-1) z}{1+z} .
$$

Using Theorem 6, we have

$$
\begin{aligned}
& \left(\mathscr{D}_{\lambda_{1}, \lambda_{2}, \delta}^{m, b} f(z) * \varphi_{\lambda_{2}}^{b}(z)\right)^{\prime} \\
& \quad \prec q(z) \\
& \quad=2 \alpha-1+\frac{2(1-\alpha)(1+b)}{\left(\lambda_{1}+\lambda_{2}\right) z^{(1+b) /\left(\lambda_{1}+\lambda_{2}\right)}} \sigma\left(\frac{1+b}{\lambda_{1}+\lambda_{2}}\right) .
\end{aligned}
$$

Since $q$ is convex and $q(\mathbb{U})$ is symmetric with respect to the real axis, we deduce that

$$
\begin{aligned}
\mathfrak{R}\left(\mathscr{D}_{\lambda_{1}, \lambda_{2}, \delta}^{m, b} f(z) * \varphi_{\lambda_{2}}^{b}(z)\right)^{\prime} \\
\quad>\Re_{q}(1) \\
\quad=\beta\left(\alpha, \lambda_{1}, \lambda_{2}, b\right) \\
\quad=2 \alpha-1+\frac{2(1-\alpha)(1+b)}{\left(\lambda_{1}+\lambda_{2}\right)} \sigma\left(\frac{1+b}{\lambda_{1}+\lambda_{2}}\right),
\end{aligned}
$$

for which we deduce $\mathscr{R}_{\lambda_{1}, \lambda_{2}, \delta}^{m+1, b}(\alpha) \subset \mathscr{K}_{\lambda_{1}, \lambda_{2}, \delta}^{m, b}(\beta)$. The proof is complete. 
Theorem 8. Let $q$ be a convex function in $\mathbb{U}$, with $q(0)=1$, and let

$$
h(z)=q(z)+\frac{\left(\lambda_{1}+\lambda_{2}\right)}{1+b} z q^{\prime}(z), \quad(z \in \mathbb{U}) .
$$

If $\lambda_{2} \geq \lambda_{1} \geq 0, \delta, m, b \in \mathbb{N}_{0}$, and $f \in \mathscr{A}$ satisfies the differential subordination

$$
\left(\mathscr{D}_{\lambda_{1}, \lambda_{2}, \delta}^{m+1, b} f(z)\right)^{\prime} \prec h(z)
$$

then

$$
\left(\mathscr{D}_{\lambda_{1}, \lambda_{2}, \delta}^{m, b} f(z) * \varphi_{\lambda_{2}}^{b}(z)\right)^{\prime} \prec q(z), \quad(z \in \mathbb{U}),
$$

and the result is sharp.

Proof. Using (28) in (26), the differential subordination (39) becomes

$$
\begin{aligned}
p(z) & +\frac{\left(\lambda_{1}+\lambda_{2}\right)}{1+b} z p^{\prime}(z) \\
& \prec h(z)=q(z)+\frac{\left(\lambda_{1}+\lambda_{2}\right)}{1+b} z q^{\prime}(z) \quad(z \in \mathbb{U}) .
\end{aligned}
$$

Using Lemma 2, we have

$$
p(z) \prec q(z), \quad(z \in \mathbb{U}) ;
$$

that is,

$$
\left(\mathscr{D}_{\lambda_{1}, \lambda_{2}, \delta}^{m, b} f(z) * \varphi_{\lambda_{2}}^{b}(z)\right)^{\prime} \prec q(z), \quad(z \in \mathbb{U}),
$$

and the result is sharp. The proof of Theorem 8 is complete.

Example 9. For $m=1, \delta=0, \lambda_{2} \geq \lambda_{1} \geq 0, b \in \mathbb{N}_{0}, q(z)=$ $(1+z) /(1-z), f \in \mathscr{A}$, and $z \in \mathbb{U}$, by applying Theorem 8 , we have

$$
\begin{aligned}
h(z) & =\frac{1+z}{1-z}+\frac{\left(\lambda_{1}+\lambda_{2}\right)}{1+b} z\left(\frac{1+z}{1-z}\right)^{\prime} \\
& =\frac{(1+b)+2\left(\lambda_{1}+\lambda_{2}\right) z-(1+b) z^{2}}{(1+b)(1-z)^{2}} .
\end{aligned}
$$

By using equality (18) we find that

$$
\begin{aligned}
(1+b) & \mathscr{D}_{\lambda_{1}, \lambda_{2}, 0}^{1, b} \\
= & \left(1-\left(\lambda_{1}+\lambda_{2}\right)+b\right) \\
& \times\left(f(z) * \varphi_{\lambda_{2}}^{b}(z)\right)+\left(\lambda_{1}+\lambda_{2}\right) z\left(f(z) * \varphi_{\lambda_{2}}^{b}(z)\right)^{\prime} .
\end{aligned}
$$

Now,

$$
\begin{aligned}
& (1+b)\left(\mathscr{D}_{\lambda_{1}, \lambda_{2}, 0}^{1, b} * \varphi_{\lambda_{2}}^{b}(z)\right) \\
& =\left(1-\left(\lambda_{1}+\lambda_{2}\right)+b\right)\left(z+\sum_{n=2}^{\infty} \frac{a_{n} z^{n}}{\left(1+\lambda_{2}(n-1)+b\right)^{2}}\right) \\
& \quad+\left(\lambda_{1}+\lambda_{2}\right)\left(z+\sum_{n=2}^{\infty} \frac{n a_{n} z^{n}}{\left(1+\lambda_{2}(n-1)+b\right)^{2}}\right) .
\end{aligned}
$$

A straightforward calculation gives the following:

$$
\begin{aligned}
& \left(\mathscr{D}_{\lambda_{1}, \lambda_{2}, 0}^{1, b} f(z) * \varphi_{\lambda_{2}}^{b}(z)\right)^{\prime} \\
& =1+\sum_{n=2}^{\infty}\left(\frac{1+\left(\lambda_{1}+\lambda_{2}\right)(n-1)+b}{\left(1+\lambda_{2}(n-1)+b\right)^{2}}\right) n a_{n} z^{n-1} \\
& =\left(z+\sum_{n=2}^{\infty}\left(\frac{\left(n\left(1+\left(\lambda_{1}+\lambda_{2}\right)(n-1)+b\right)\right)}{\left(1+\lambda_{2}(n-1)+b\right)^{2}}\right) a_{n} z^{n}\right) \\
& \quad \times(z)^{-1} \\
& =\left(\left(f(z) * \varphi_{\lambda_{2}}^{b}(z)\right)\right. \\
& \left.\quad *\left(z+\sum_{n=2}^{\infty}\left(\frac{n\left(1+\left(\lambda_{1}+\lambda_{2}\right)(n-1)+b\right)}{\left(1+\lambda_{2}(n-1)+b\right)}\right) a_{n} z^{n}\right)\right) \\
& \quad \times(z)^{-1} .
\end{aligned}
$$

Similarly, using (18), we see that

$$
\begin{aligned}
(1+b) & \mathscr{D}_{\lambda_{1}, \lambda_{2}, 0}^{2, b} f(z) \\
= & \left(1-\left(\lambda_{1}+\lambda_{2}\right)+b\right) \\
& \times\left(\mathscr{D}_{\lambda_{1}, \lambda_{2}, 0}^{1, b} f(z) * \varphi_{\lambda_{2}}^{b}(z)\right) \\
& +\left(\lambda_{1}+\lambda_{2}\right) z\left(\mathscr{D}_{\lambda_{1}, \lambda_{2}, 0}^{1, b} f(z) * \varphi_{\lambda_{2}}^{b}(z)\right)^{\prime},
\end{aligned}
$$

and then

$$
\begin{aligned}
(1+b) & \left(\mathscr{D}_{\lambda_{1}, \lambda_{2}, 0}^{2, b} f(z)\right)^{\prime} \\
= & (1+b)\left(\mathscr{D}_{\lambda_{1}, \lambda_{2}, 0}^{1, b} f(z) * \varphi_{\lambda_{2}}^{b}(z)\right)^{\prime} \\
& +\left(\lambda_{1}+\lambda_{2}\right) z\left(\mathscr{D}_{\lambda_{1}, \lambda_{2}, 0}^{1, b} f(z) * \varphi_{\lambda_{2}}^{b}(z)\right)^{\prime \prime} .
\end{aligned}
$$

By using (47) we have

$$
\begin{aligned}
& \left(\mathscr{D}_{\lambda_{1}, \lambda_{2}, 0}^{1, b} f(z) * \varphi_{\lambda_{2}}^{b}(z)\right)^{\prime \prime} \\
& \quad=\sum_{n=2}^{\infty}\left(\frac{1+\left(\lambda_{1}+\lambda_{2}\right)(n-1)+b}{\left(1+\lambda_{2}(n-1)+b\right)^{2}}\right) n(n-1) a_{n} z^{n-2} ;
\end{aligned}
$$

we deduce

$$
\begin{aligned}
& (1+b)\left(\mathscr{D}_{\lambda_{1}, \lambda_{2}, 0}^{2, b} f(z)\right)^{\prime} \\
& =(1+b)\left(1+\sum_{n=2}^{\infty}\left(\frac{1+\left(\lambda_{1}+\lambda_{2}\right)(n-1)+b}{\left(1+\lambda_{2}(n-1)+b\right)^{2}}\right) n a_{n} z^{n-1}\right) \\
& \quad+\left(\lambda_{1}+\lambda_{2}\right) \\
& \quad \times \sum_{n=2}^{\infty}\left(\frac{1+\left(\lambda_{1}+\lambda_{2}\right)(n-1)+b}{\left(1+\lambda_{2}(n-1)+b\right)^{2}}\right) n(n-1) a_{n} z^{n-1}
\end{aligned}
$$


that is,

$$
\begin{aligned}
& \left(\mathscr{D}_{\lambda_{1}, \lambda_{2}, 0}^{2, b} f(z)\right)^{\prime} \\
& =1+\sum_{n=2}^{\infty}\left(\frac{1+\left(\lambda_{1}+\lambda_{2}\right)(n-1)+b}{1+\lambda_{2}(n-1)+b}\right)^{2} n a_{n} z^{n-1} \\
& =\left(\left(f(z) * \varphi_{\lambda_{2}}^{b}(z)\right)\right. \\
& \left.\quad *\left(z+\sum_{n=2}^{\infty}\left(\frac{n\left(1+\left(\lambda_{1}+\lambda_{2}\right)(n-1)+b\right)^{2}}{\left(1+\lambda_{2}(n-1)+b\right)}\right) a_{n} z^{n}\right)\right) \\
& \quad \times(z)^{-1} .
\end{aligned}
$$

From Theorem 8 we get

$$
\begin{aligned}
& \left(\left(f(z) * \varphi_{\lambda_{2}}^{b}(z)\right)\right. \\
& \left.*\left(z+\sum_{n=2}^{\infty}\left(\frac{n\left(1+\left(\lambda_{1}+\lambda_{2}\right)(n-1)+b\right)^{2}}{\left(1+\lambda_{2}(n-1)+b\right)}\right) a_{n} z^{n}\right)\right) \times(z)^{-1} \\
& \quad \prec \frac{(1+b)+2\left(\lambda_{1}+\lambda_{2}\right) z-(1+b) z^{2}}{(1+b)(1-z)^{2}},
\end{aligned}
$$

which implies that

$$
\begin{aligned}
& \left(\left(f(z) * \varphi_{\lambda_{2}}^{b}(z)\right)\right. \\
& \left.\quad *\left(z+\sum_{n=2}^{\infty}\left(\frac{n\left(1+\left(\lambda_{1}+\lambda_{2}\right)(n-1)+b\right)}{\left(1+\lambda_{2}(n-1)+b\right)}\right) a_{n} z^{n}\right)\right) \times(z)^{-1} \\
& \quad \prec \frac{1+z}{1-z}, \quad(z \in \mathbb{U}) .
\end{aligned}
$$

Theorem 10. Let $q$ be a convex function in $\mathbb{U}$, with $q(0)=1$, and let

$$
h(z)=q(z)+z q^{\prime}(z), \quad(z \in \mathbb{U}) .
$$

If $\lambda_{2} \geq \lambda_{1} \geq 0, \delta, m, b \in \mathbb{N}_{0}$, and $f \in \mathscr{A}$ satisfies the differential subordination

$$
\left(\mathscr{D}_{\lambda_{1}, \lambda_{2}, \delta}^{m, b} f(z)\right)^{\prime} \prec h(z)
$$

then

$$
\frac{\mathscr{D}_{\lambda_{1}, \lambda_{2}, \delta}^{m, b} f(z)}{z} \prec q(z), \quad(z \in \mathbb{U}),
$$

and the result is sharp.
Proof. Let

$$
p(z)=\frac{\mathscr{D}_{\lambda_{1}, \lambda_{2}, \delta}^{m, b} f(z)}{z}, \quad(z \in \mathbb{U}) .
$$

Differentiating (58), with respect to $z$, we obtain

$$
\left(\mathscr{D}_{\lambda_{1}, \lambda_{2}, \delta}^{m, b} f(z)\right)^{\prime}=p(z)+z p^{\prime}(z), \quad(z \in \mathbb{U}) .
$$

Using (58), the differential subordination (56) becomes $p(z)+z p^{\prime}(z) \prec h(z)=q(z)+z q^{\prime}(z), \quad(z \in \mathbb{U})$.

Using Lemma 2, we deduce that

$$
p(z) \prec q(z), \quad(z \in \mathbb{U}) .
$$

By using (58), we have

$$
\frac{\mathscr{D}_{\lambda_{1}, \lambda_{2}, \delta}^{m, b} f(z)}{z} \prec q(z), \quad(z \in \mathbb{U}) .
$$

The proof of Theorem 10 is complete.

Example 11. For $\delta=0, m=1, \lambda_{2} \geq \lambda_{1} \geq 0, b \in \mathbb{N}_{0}, q(z)=$ $1 /(1-z), f \in \mathscr{A}$, and $z \in \mathbb{U}$, from Theorem 10 we obtain

$$
h(z)=\frac{1}{1-z}+z\left(\frac{1}{1-z}\right)^{\prime}=\frac{1}{(1-z)^{2}}
$$

From Example 9, we have

$$
\begin{aligned}
(1+b) & \mathscr{D}_{\lambda_{1}, \lambda_{2}, 0}^{1, b} f(z) \\
= & \left(1-\left(\lambda_{1}+\lambda_{2}\right)+b\right)\left(f(z) * \varphi_{\lambda_{2}}^{b}\right) \\
& +\left(\lambda_{1}+\lambda_{2}\right) z\left(f(z) * \varphi_{\lambda_{2}}^{b}\right)^{\prime},
\end{aligned}
$$

and then

$$
\begin{aligned}
(1+b)\left(\mathscr{D}_{\lambda_{1}, \lambda_{2}, 0}^{1, b} f(z)\right)^{\prime}= & (1+b)\left(f(z) * \varphi_{\lambda_{2}}^{b}\right)^{\prime} \\
& +\left(\lambda_{1}+\lambda_{2}\right) z\left(f(z) * \varphi_{\lambda_{2}}^{b}\right)^{\prime \prime} .
\end{aligned}
$$

From Theorem 10 we deduce that

$$
\left(f(z) * \varphi_{\lambda_{2}}^{b}\right)^{\prime}+\frac{\left(\lambda_{1}+\lambda_{2}\right)}{(1+b)} z\left(f(z) * \varphi_{\lambda_{2}}^{b}\right)^{\prime \prime} \prec \frac{1}{(1-z)^{2}}
$$

implies that

$$
\frac{\left(1-\left(\lambda_{1}+\lambda_{2}\right)+b\right)\left(f(z) * \varphi_{\lambda_{2}}^{b}\right)+\left(\lambda_{1}+\lambda_{2}\right) z\left(f(z) * \varphi_{\lambda_{2}}^{b}\right)^{\prime}}{z(1+b)}
$$


Theorem 12. Let $h$ be a convex function in $\mathbb{U}$, with $h(0)=$ $1,0 \leq \alpha<1$, and let

$$
h(z)=\frac{1+(2 \alpha-1) z}{1+z}, \quad(z \in \mathbb{U}) .
$$

If $\lambda_{2} \geq \lambda_{1} \geq 0, \delta, m, b \in \mathbb{N}_{0}$, and $f \in \mathscr{A}$ satisfies the differential subordination

$$
\left(\mathscr{D}_{\lambda_{1}, \lambda_{2}, \delta}^{m, b} f(z)\right)^{\prime} \prec h(z)
$$

then

$$
\begin{aligned}
\frac{\mathscr{D}_{\lambda_{1}, \lambda_{2}, \delta}^{m, b} f(z)}{z} \prec q(z)= & 2 \alpha-1 \\
& +\frac{2(1-\alpha) \ln (1+z)}{z}, \quad(z \in \mathbb{U}) .
\end{aligned}
$$

The function $q$ is convex and is the best dominant.

Proof. Let

$$
\begin{aligned}
p(z)= & \frac{\mathscr{D}_{\lambda_{1}, \lambda_{2}, \delta}^{m, b} f(z)}{z} \\
= & \left(z+\sum_{n=2}^{\infty}\left(\frac{\left(1+\left(\lambda_{1}+\lambda_{2}\right)(n-1)+b\right)^{m}}{\left(1+\lambda_{2}(n-1)+b\right)^{m}}\right)\right. \\
& \left.\times \mathscr{C}(\delta, n) a_{n} z^{n}\right) \times(z)^{-1} \\
= & 1+p_{1} z+p_{2} z^{2}+\cdots, \quad(p \in \mathscr{H}[1,1], \quad z \in \mathbb{U}) .
\end{aligned}
$$

Differentiating (71), with respect to $z$, we obtain

$$
\left(\mathscr{D}_{\lambda_{1}, \lambda_{2}, \delta}^{m, b} f(z)\right)^{\prime}=p(z)+z p^{\prime}(z), \quad(z \in \mathbb{U}) .
$$

Using (72), the differential subordination (69) becomes

$$
p(z)+z p^{\prime}(z) \prec h(z)=\frac{1+(2 \alpha-1) z}{1+z}, \quad(z \in \mathbb{U}) .
$$

Using Lemma 1, we deduce that

$$
\begin{aligned}
p(z) \prec q(z) & =\frac{1}{z} \int_{0}^{z} h(t) d t \\
& =\frac{1}{z} \int_{0}^{z}\left(\frac{1+(2 \alpha-1) t}{1+t}\right) d t \\
& =\frac{1}{z}\left(\int_{0}^{z} \frac{1}{1+t} d t+(2 \alpha-1) \int_{0}^{z} \frac{t}{1+t} d t\right) \\
& =2 \alpha-1+\frac{2(1-\alpha) \ln (1+z)}{z} .
\end{aligned}
$$

By using (71), we have

$$
\frac{\mathscr{D}_{\lambda_{1}, \lambda_{2}, \delta}^{m, b} f(z)}{z} \prec q(z)=2 \alpha-1+\frac{2(1-\alpha) \ln (1+z)}{z} .
$$

The proof of Theorem 12 is complete.
Corollary 13. If $f \in \mathscr{R}_{\lambda_{1}, \lambda_{2}, \delta}^{m, b}(\alpha)$, then

$$
\mathfrak{R}\left(\frac{\mathscr{D}_{\lambda_{1}, \lambda_{2}, \delta}^{m, b} f(z)}{z}\right)>(2 \alpha-1)+2(1-\alpha) \ln 2, \quad(z \in \mathbb{U}) .
$$

Proof. Since $f \in \mathscr{R}_{\lambda_{1}, \lambda_{2}, \delta}^{m, b}(\alpha)$, from Definition 4 we have

$$
\mathfrak{R}\left(\mathscr{D}_{\lambda_{1}, \lambda_{2}, \delta}^{m, b}(\alpha)\right)^{\prime}>\alpha, \quad(z \in \mathbb{U}),
$$

which is equivalent to

$$
\left(\mathscr{D}_{\lambda_{1}, \lambda_{2}, \delta}^{m, b}(\alpha)\right)^{\prime} \prec h(z)=\frac{1+(2 \alpha-1) z}{1+z} .
$$

Using Theorem 12, we obtain

$$
\frac{\mathscr{D}_{\lambda_{1}, \lambda_{2}, \delta}^{m, b} f(z)}{z} \prec q(z)=2 \alpha-1+\frac{2(1-\alpha) \ln (1+z)}{z} .
$$

Since $q$ is convex and $q(\mathbb{U})$ is symmetric with respect to the real axis, we have that

$$
\begin{aligned}
\mathfrak{R}\left(\frac{\mathscr{D}_{\lambda_{1}, \lambda_{2}, \delta}^{m, b} f(z)}{z}\right)> & \Re q(1)=(2 \alpha-1) \\
& +2(1-\alpha) \ln 2, \quad(z \in \mathbb{U}) .
\end{aligned}
$$

Theorem 14. Let $h \in \mathscr{H}(\mathbb{U})$, with $h(0)=1, h^{\prime}(0) \neq 0$, which satisfies the inequality

$$
\Re\left(1+\frac{z h^{\prime \prime}(z)}{h^{\prime}(z)}\right)>-\frac{1}{2}, \quad(z \in \mathbb{U}) .
$$

If $\lambda_{2} \geq \lambda_{1} \geq 0, \delta, m, b \in \mathbb{N}_{0}$, and $f \in \mathscr{A}$ satisfies the differential subordination

$$
\left(\mathscr{D}_{\lambda_{1}, \lambda_{2}, \delta}^{m, b} f(z)\right)^{\prime} \prec h(z),
$$

then

$$
\frac{\mathscr{D}_{\lambda_{1}, \lambda_{2}, \delta}^{m, b} f(z)}{z} \prec q(z)=\frac{1}{z} \int_{0}^{z} h(t) d t .
$$

Proof. Let

$$
\begin{aligned}
p(z)= & \frac{\mathscr{D}_{\lambda_{1}, \lambda_{2}, \delta}^{m, b} f(z)}{z} \\
= & \left(z+\sum_{n=2}^{\infty}\left(\frac{\left(1+\left(\lambda_{1}+\lambda_{2}\right)(n-1)+b\right)^{m}}{\left(1+\lambda_{2}(n-1)+b\right)^{m}}\right)\right. \\
& \left.\quad \times \mathscr{C}(\delta, n) a_{n} z^{n}\right) \times(z)^{-1} \\
= & 1+p_{1} z+p_{2} z^{2}+\cdots, \quad(p \in \mathscr{H}[1,1], \quad z \in \mathbb{U}) .
\end{aligned}
$$


Differentiating (84), with respect to $z$, we obtain

$$
\left(\mathscr{D}_{\lambda_{1}, \lambda_{2}, \delta}^{m, b} f(z)\right)^{\prime}=p(z)+z p^{\prime}(z), \quad(z \in \mathbb{U}) .
$$

Using (85), the differential subordination (82) becomes

$$
p(z)+z p^{\prime}(z) \prec h(z), \quad(z \in \mathbb{U}) .
$$

Using Lemma 1, we deduce that

$$
p(z) \prec q(z)=\frac{1}{z} \int_{0}^{z} h(t) d t
$$

by using (84), we have

$$
\frac{\mathscr{D}_{\lambda_{1}, \lambda_{2}, \delta}^{m, b} f(z)}{z} \prec q(z)=\frac{1}{z} \int_{0}^{z} h(t) d t .
$$

From Lemma 3, we see that the function $q$ is convex, and from Lemma $1, q$ is the best dominant for subordination (82). The proof of Theorem 14 is complete.

Note that other work related to differential operators and differential subordination can be seen in [8-13].

\section{Conflict of Interests}

The authors declare that there is no conflict of interests regarding the publication of this paper.

\section{Authors' Contribution}

Entisar El-Yagubi and Maslina Darus read and approved the final paper.

\section{Acknowledgment}

The work presented here was partially supported by LRGS/TD/2013/UKM/ICT/03/02.

\section{References}

[1] D. J. Hallenbeck and S. Ruscheweyh, "Subordination by convex functions," Proceedings of the American Mathematical Society, vol. 52, pp. 191-195, 1975.

[2] S. S. Miller and P. T. Mocanu, Differential Subordinations. Theory and Applications, vol. 225 of Monographs and Textbooks in Pure and Applied Mathematics, Marcel Dekker, New York, NY, USA, 2000.

[3] S. S. Miller and P. T. Mocanu, "On some classes of firstorder differential subordinations," The Michigan Mathematical Journal, vol. 32, no. 2, pp. 185-195, 1985.

[4] E. El-Yagubi and M. Darus, "Subclasses of analytic functions defined by new generalised derivative operator," Journal of Quality Measurement and Analysis, vol. 9, no. 1, pp. 47-56, 2013.

[5] G. I. Oros, "A class of holomorphic functions defined using a differential operator," General Mathematics, vol.13, no. 4, pp.1318, 2005.

[6] G. Oros and G. I. Oros, "Differential superordination defined by Salagean operator," General Mathematics, vol. 12, no. 4, pp. 3-10, 2004.
[7] S. Ponnusamy, "Differential subordination and starlike functions," Complex Variables. Theory and Application, vol. 19, no. 3, pp. 185-194, 1992.

[8] M. Darus and K. Al-Shaqsi, "Differential sandwich theorems with generalised derivative operator," International Journal Mathematical Sciences, vol. 2, no. 2, pp. 75-78, 2008.

[9] R. W. Ibrahim and M. Darus, "Subordination and superordination for univalent solutions for fractional differential equations," Journal of Mathematical Analysis and Applications, vol. 345, no. 2, pp. 871-879, 2008.

[10] O. Al-Refai and M. Darus, "Main differential sandwich theorem with some applications," Lobachevskii Journal of Mathematics, vol. 30, no. 1, pp. 1-11, 2009.

[11] B. A. Frasin and M. Darus, "Subordination results on subclasses concerning Sakaguchi functions," Journal of Inequalities and Applications, vol. 2009, Article ID 574014, 7 pages, 2009.

[12] M. H. Al-Abbadi and M. Darus, "Differential subordination for new generalised derivative operator," Acta Universitatis Apulensis., no. 20, pp. 265-280, 2009.

[13] M. Harayzeh Al-Abbadi and M. Darus, "Differential subordination defined by new generalised derivative operator for analytic functions," International Journal of Mathematics and Mathematical Sciences, vol. 2010, Article ID 369078, 15 pages, 2010. 


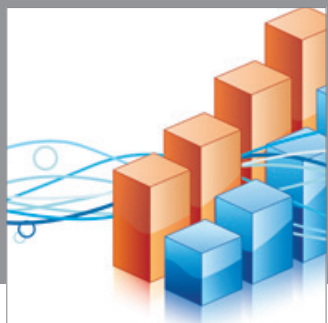

Advances in

Operations Research

mansans

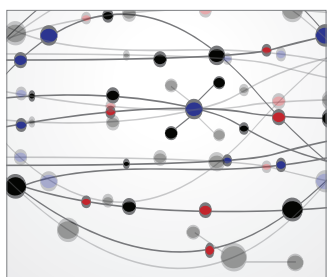

The Scientific World Journal
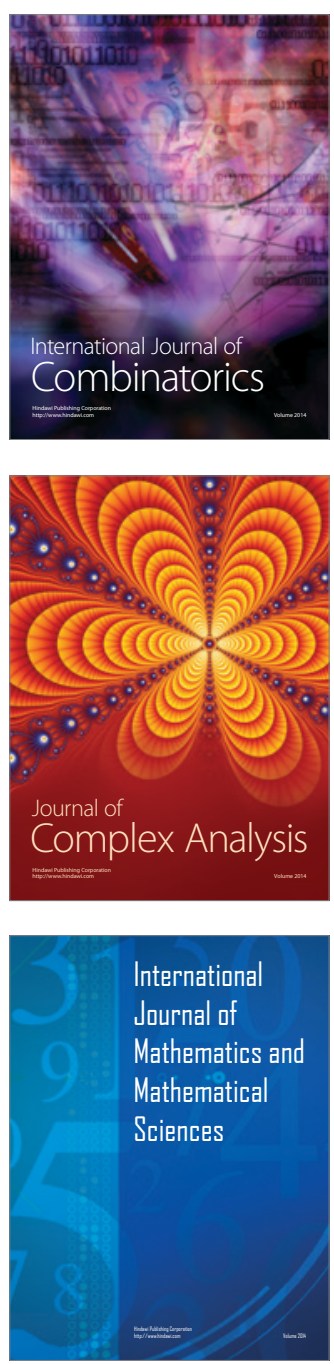
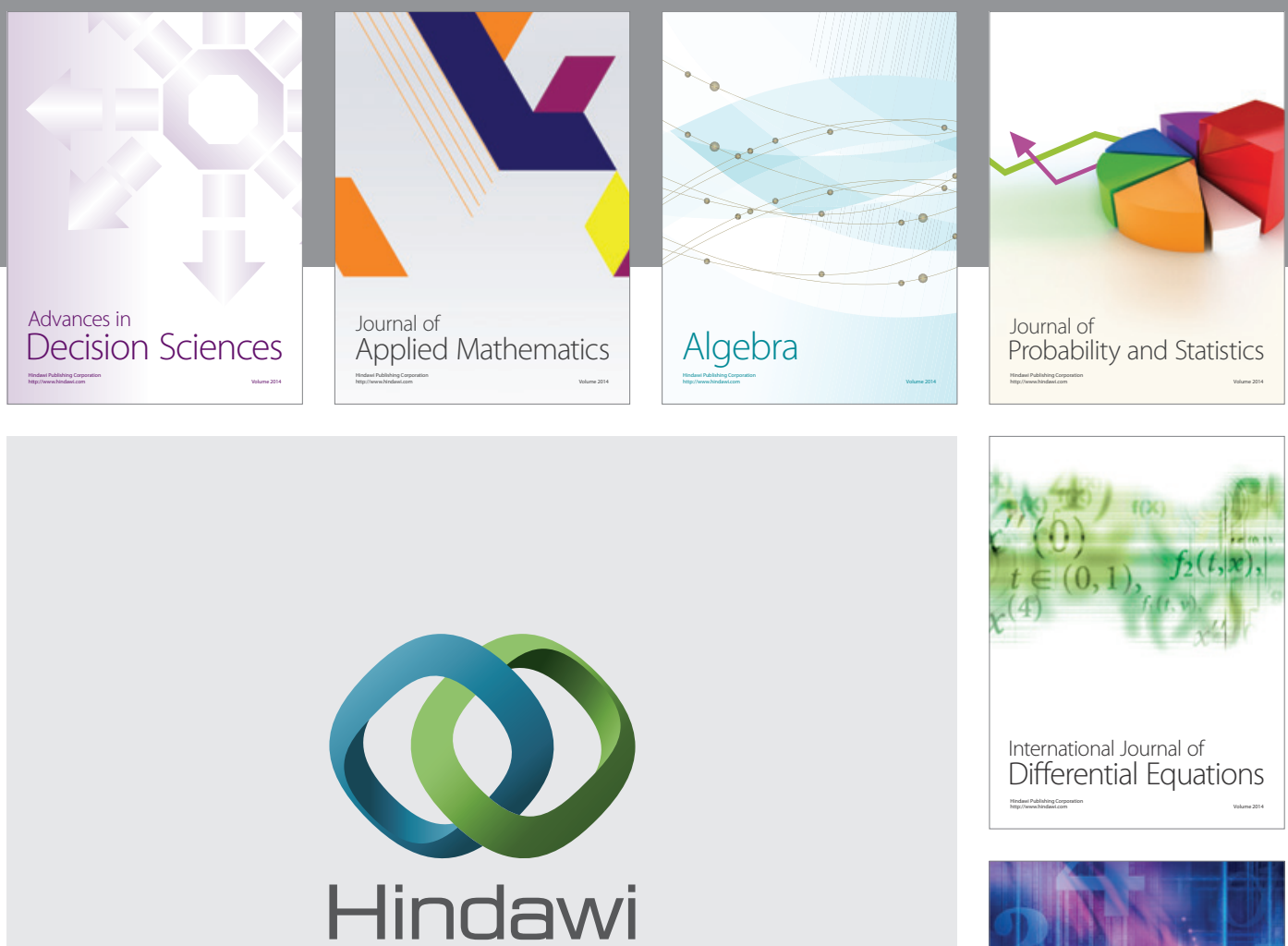

Submit your manuscripts at http://www.hindawi.com
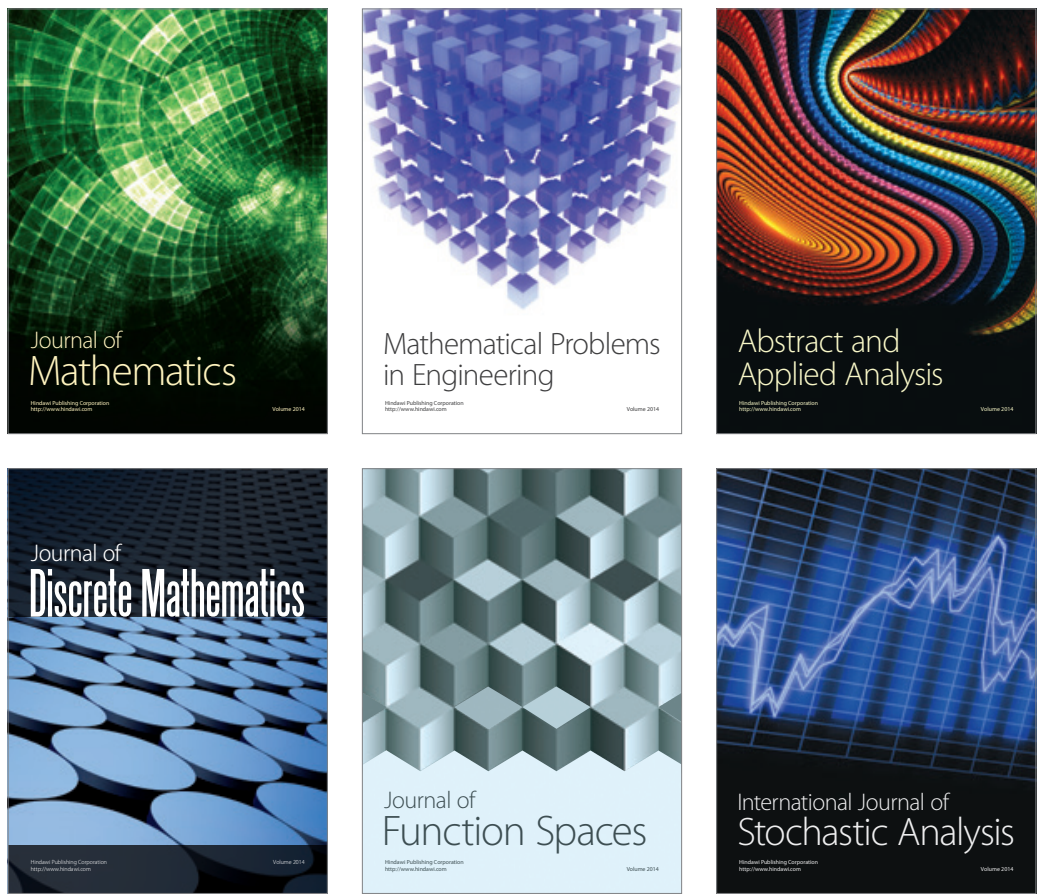

Journal of

Function Spaces

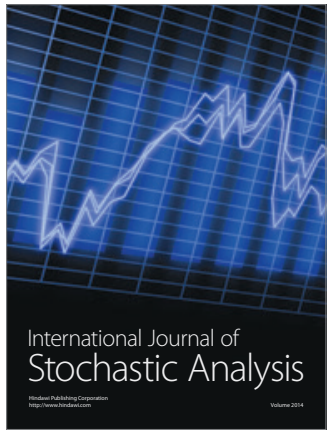

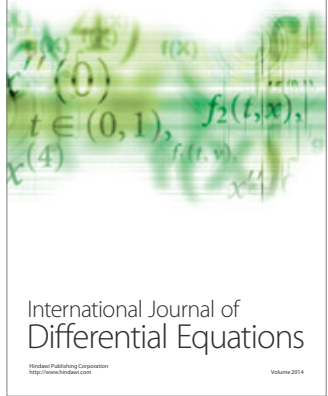
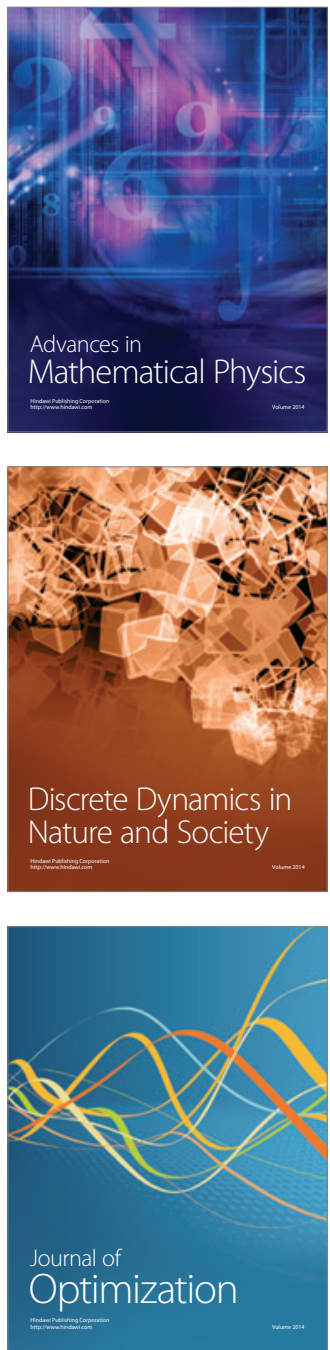